\title{
The Hölder continuity of a class of 3-dimension ultraparabolic equations
}

\author{
WANG Wendong and ZHANG Liqun * \\ Institute of Mathematics, AMSS, Academia Sinica, Beijing
}

\begin{abstract}
We obtained the $C^{\alpha}$ continuity for weak solutions of a class of ultraparabolic equations with measurable coefficients of the form$$
\partial_{t} u=\partial_{x}\left(a(x, y, t) \partial_{x} u\right)+b_{0}(x, y, t) \partial_{x} u+b(x, y, t) \partial_{y} u,
$$

which generalized our recent results on KFP equations.

keywords: Hypoelliptic, ultraparabolic equations, Hölder regularity

\section{Introduction}

Consider a class of ultraparabolic operator on $R^{2+1}$ :

$$
L u \equiv \partial_{x}\left(a(x, y, t) \partial_{x} u\right)+b_{0}(x, y, t) \partial_{x} u+b(x, y, t) \partial_{y} u-\partial_{t} u=0,
$$

where $(x, y, t)=z \in \Omega \subset R^{2+1}, a(z), b_{0}(z)$ and $b(z)$ is real, measurable functions. We assume that $b(z)$ is twice differentiable, and there exists a positive constant $\mu$ such that for $z \in \Omega$,

$$
\mu<a(z)<\mu^{-1}, \quad \frac{\partial b(z)}{\partial x} \neq 0, \quad|b|_{C^{2}}+\left|b_{0}\right|_{\infty} \leq \mu^{-1} .
$$

${ }^{*}$ The research is partially supported by the Chinese NSF under grant 10325104. Email: wangwendong@amss.ac.cn and lqzhang@math.ac.cn 
Also, we denote

$$
\begin{gathered}
L_{0} u=\partial_{x}^{2} u+x \partial_{y} u-\partial_{t} u=0, \\
L_{1} u=\partial_{x}\left(a(x, y, t) \partial_{x} u\right)+x \partial_{y} u-\partial_{t} u=0,
\end{gathered}
$$

and

$$
L_{2} u=\partial_{x}\left(a(x, y, t) \partial_{x} u\right)+b_{0}(x, y, t) \partial_{x} u+x \partial_{y} u-\partial_{t} u=0
$$

We remark that the equation (1.3) and (1.4) are the examples of 3dimension homogeneous Kolmogorov-Fokker-Planck equations (or KFP equations). The condition $\frac{\partial b}{\partial x} \neq 0$ ensures (1.1) satisfies Hörmander's hypoellipticity conditions,

$$
\operatorname{rank} \operatorname{Lie}\left(\partial_{x}, b \partial_{y}-\partial_{t}\right)(z)=3, \quad \forall z \in \Omega
$$

The study of regularity of the KFP equation has a long history, and the earlier works are mainly on the Schauder type estimates. The study of regularity of weak solutions is begun in recent years. A recent paper of Pascucci and Polidoro [6], has proved that the Moser iterative method still works for the class of KFP equations with measurable coefficients. By the same technique, Cinti, Pascucci, Polidoro [1] consider a class of nonhomogeneous KFP equations, and Cinti, Polidoro [2] deal with a more general ultraparabolic equation. Their results show that for a non-negative sub-solution $u$ of the ultraparabolic equation, $L^{\infty}$ norm of $u$ is bounded by the $L^{p}$ norm $(p \geq 1)$. The second author [10], [11] has proved $C^{\alpha}$ property of weak solutions by Kruzhkov's approach for homogeneous KFP equations, and the authors deal with nonhomogeneous KFP equations in [7]. By simplifying the cut-off function and generalizing their earlier arguments, the authors [8] have considered more general ultraparabolic equations whose fundamental solution is implicit. 
We are not try to review the detailed history, but focus on the study of the Hölder continuity of a simple looking case. In this paper, we give another generalization of KFP equations in $R^{2+1}$ and consider the hypoelliptic operator as $\mathrm{L}$ in (1.1).

We say that $u$ is a weak solution if it satisfies (1.1) in the distribution sense, that is for any $\phi \in C_{0}^{\infty}(\Omega), \Omega$ is a open subset of $R^{2+1}$, then

$$
\int_{\Omega} \phi\left(b_{0} \partial_{x}+b \partial_{y}-\partial_{t}\right) u-a \partial_{x} \phi \partial_{x} u=0
$$

and $u, \partial_{x} u, b \partial_{y}-\partial_{t} u \in L_{\mathrm{loc}}^{2}(\Omega)$.

Our main result is the following theorem:

Theorem 1.1 Under the assumption (1.2), the weak solution of (1.1) is Hölder continuous.

\section{Some Preliminary and Known Results}

We follow the earlier notations to give some basic known properties related to our problems. For more details of the subject, we refer to Pascucci and Polidoro [6] and Lanconelli and Polidoro [5].

$$
\begin{aligned}
& \text { Let } B=\left(\begin{array}{ll}
0 & 1 \\
0 & 0
\end{array}\right) \text {, and } E(\tau)=\exp \left(-\tau \mathrm{B}^{\mathrm{T}}\right)=\left(\begin{array}{cc}
1 & 0 \\
-\tau & 1
\end{array}\right) . \\
& \text { For }(x, y, t),(\xi, \eta, \tau) \in R^{2+1}, \text { set } \\
& \qquad(x, y, t) \circ(\xi, \eta, \tau)=\left((\xi, \eta)+E(\tau)\left(\begin{array}{l}
x \\
y
\end{array}\right), t+\tau\right),
\end{aligned}
$$

then $\left(R^{2+1}, \circ\right)$ is a Lie group with identity element $(0,0)$, and the inverse of an element is $(x, y, t)^{-1}=\left(-E(-t)\left(\begin{array}{l}x \\ y\end{array}\right),-t\right)$. The left translation by $(\xi, \eta, \tau)$ given by

$$
(x, y, t) \mapsto(\xi, \eta, \tau) \circ(x, y, t)
$$


is a invariant translation to the operator $L_{0}$. The associated dilation to operator $L_{0}$ is given by

$$
\delta_{t}=\operatorname{diag}\left(t, t^{3}, t^{2}\right)
$$

where $t$ is a positive parameter, and the homogeneous dimension of $\left(R^{2+1}, \circ\right)$ with respect to the dilation $\delta_{t}$ is 6 .

The norm in $R^{2+1}$, related to the group of translations and dilation to the equation is defined by $\|(x, y, t)\|=r$, if $r$ is the unique positive solution to the equation

$$
\frac{x^{2}}{r^{2}}+\frac{y^{2}}{r^{6}}+\frac{t^{2}}{r^{4}}=1
$$

where $(x, y, t) \in R^{2+1} \backslash\{0\}$. And $\|(0,0)\|=0$. Obviously

$$
\left\|\delta_{\mu}(x, y, t)\right\|=\mu\|(x, y, t)\|
$$

for all $(x, y, t) \in R^{2+1}$.

The ball centered at a point $\left(x_{0}, t_{0}\right)$ is defined by

$$
\mathcal{B}_{r}\left(x_{0}, t_{0}\right)=\left\{(x, t) \mid \quad\left\|\left(x_{0}, t_{0}\right)^{-1} \circ(x, t)\right\| \leq r\right\}
$$

and

$$
\mathcal{B}_{r}^{-}\left(x_{0}, t_{0}\right)=\mathcal{B}_{r}\left(x_{0}, t_{0}\right) \cap\left\{t<t_{0}\right\} .
$$

For convenience, we sometimes use the cube instead of the balls. The cube at point $(0,0)$ is given by

$$
\mathcal{C}_{r}(0,0)=\left\{(x, y, t)|\quad| x|\leq r,| y\left|\leq 8 r^{3}, \quad\right| t \mid \leq r^{2}\right\}
$$

It is easy to see that there exists a constant $\Lambda$ such that

$$
\mathcal{C}_{\frac{r}{\Lambda}}(0,0) \subset \mathcal{B}_{r}(0,0) \subset \mathcal{C}_{\Lambda r}(0,0)
$$

For $L_{0}$, the fundamental solution $\Gamma(\cdot, \zeta)$ with pole in $\zeta=(\xi, \eta, \tau) \in R^{2+1}$ is smooth except at the diagonal of $R^{2+1} \times R^{2+1}$. It has the following form 
at $\zeta=0$,

$$
\Gamma(z)=\Gamma(z, 0)=\left\{\begin{array}{cc}
\frac{\sqrt{3}}{2 \pi t^{2}} \exp \left[-\frac{1}{t}\left(x^{2}+\frac{3}{t} x y+\frac{3}{t^{2}} y^{2}\right)\right] & \text { if } t>0 \\
0 & \text { if } \quad t \leq 0
\end{array}\right.
$$

and

$$
\Gamma(z, \zeta)=\left\{\begin{array}{cl}
\frac{\sqrt{3}}{2 \pi(t-\tau)^{2}} \exp \left[-\frac{x^{2}+x \xi+\xi^{2}}{t-\tau}-\frac{3(x+\xi)(y-\eta)}{(t-\tau)^{2}}-\frac{3(y-\eta)^{2}}{(t-\tau)^{3}}\right] & \text { if } t>\tau \\
0 & \text { if } t \leq \tau
\end{array}\right.
$$

Obviously we can derive from the above formula,

$$
\int_{R^{2}} \Gamma(x, y, t ; \xi, \eta, \tau) d x d y=\int_{R^{2}} \Gamma(x, y, t ; \xi, \eta, \tau) d \xi d \eta=1, \quad \text { if } \quad t>\tau,
$$

and

$$
\Gamma\left(\delta_{\mu} \circ z\right)=\mu^{-4} \Gamma(z), \quad \forall z \neq 0, \mu>0 .
$$

A weak sub-solution of (1.5) in a domain $\Omega$ is a function $u$ such that $u$, $\partial_{x} u,\left(x \partial_{y}-\partial_{t}\right) u \in L_{l o c}^{2}(\Omega)$ and for any $\phi \in C_{0}^{\infty}(\Omega), \phi \geq 0$,

$$
\int_{\Omega} \phi\left(b_{0} \partial_{x}+x \partial_{y}-\partial_{t}\right) u-\left(\partial_{x} u\right) a \partial_{x} \phi \geq 0
$$

We recall a result of Pascucci and Polidoro obtained by using the Moser's iterative method (see [6]) states as following

Lemma 2.1 Let $u$ be a non-negative weak sub-solution of (1.4) in $\Omega$. Let $\left(x_{0}, t_{0}\right) \in \Omega$ and $\overline{\mathcal{B}_{r}^{-}\left(x_{0}, t_{0}\right)} \subset \Omega$ and $p \geq 1$. Then there exists a positive constant $C$ which depends only on the operator $L$ such that, for $0<r \leq 1$

$$
\sup _{\mathcal{B}_{\frac{r}{2}}^{-}\left(x_{0}, t_{0}\right)} u^{p} \leq \frac{C}{r^{6}} \int_{\mathcal{B}_{r}^{-}\left(x_{0}, t_{0}\right)} u^{p}
$$

provided that the last integral converges. 
The second author [10] proved the following result.

Theorem 2.1 If $u$ is a weak solution of (1.4), then $u$ is Hölder continuous.

Using the same technique, we can obtain the similar result to the equation $(1.5)$.

Theorem 2.2 If $u$ is a weak solution of (1.5), then $u$ is Hölder continuous.

In section 3, we shall sketch the proof of this theorem first. We mainly focus on the proof of the oscillation estimates. Then we give a transformation as Weber in [9] and complete the proof of Theorem 1.1.

We make use of a classical potential estimates (see (1.11) in [3]) here to prove the Poincaré type inequality.

Lemma 2.2 Let $\left(R^{N+1}, \circ\right)$ is a homogeneous Lie group of homogeneous dimension $Q+2, \alpha \in(0, Q+2)$ and $G \in C\left(R^{N+1} \backslash\{0\}\right)$ be a $\delta_{\mu}$-homogeneous function of degree $\alpha-Q-2$. If $f \in L^{p}\left(R^{N+1}\right)$ for some $p \in(1, \infty)$, then

$$
G_{f}(z) \equiv \int_{R^{N+1}} G\left(\zeta^{-1} \circ z\right) f(\zeta) d \zeta,
$$

is defined almost everywhere and there exists a constant $C=C(Q, p)$ such that

$$
\left\|\left.G_{f}\right|_{L^{q}\left(R^{N+1}\right)} \leq C \max _{\|z\|=1}|G(z)| \quad\right\| f \|_{L^{p}\left(R^{N+1}\right)},
$$

where $q$ is defined by

$$
\frac{1}{q}=\frac{1}{p}-\frac{\alpha}{Q+2} .
$$

Corollary 2.1 Let $f \in L^{2}\left(R^{2+1}\right)$, and recall the definitions in [6]

$$
\Gamma(f)(z)=\int_{R^{2+1}} \Gamma(z, \zeta) f(\zeta) d \zeta, \quad \forall z \in R^{2+1},
$$


and

$$
\Gamma\left(\partial_{\xi} f\right)(z)=-\int_{R^{2+1}} \partial_{\xi} \Gamma(z, \zeta) f(\zeta) d \zeta, \quad \forall z \in R^{2+1},
$$

then exists an absolute constant $C$ such that

$$
\|\Gamma(f)\|_{L^{2 \tilde{k}\left(R^{2+1}\right)}} \leq C\|f\|_{L^{2}\left(R^{2+1}\right)},
$$

and

$$
\left\|\Gamma\left(\partial_{\xi} f\right)\right\|_{L^{2 k}\left(R^{2+1}\right)} \leq C\|f\|_{L^{2}\left(R^{2+1}\right)},
$$

where $\tilde{k}=3, k=\frac{3}{2}$.

\section{Proof of Theorem 2.2}

Outline of the proof of Theorem 2.2:

Step 1: $L^{\infty}$ estimate via Moser iteration. It can be checked that the same Caccioppoli type inequality holds ( See Theorem 3.1, [6]), since

$$
\int_{B_{1}} \psi^{2} b_{0} \partial_{x}\left(v^{2}\right) \leq \frac{1}{2} \int_{B_{1}} \psi^{2} a v^{2}+C(\mu) \int_{B_{1}} \psi^{2} v^{2} .
$$

In order to use the Moser iteration, one need to prove a Sobolev type inequality. It can be proved that the Sobolev type inequality holds for nonnegative weak sub-solution ( See Theorem 3.3 in [6]). Here one may deal

with $\int_{B_{r}}\left[\Gamma(z, \cdot) v b_{0} \partial_{x} \psi\right](\zeta)$ as $I_{2}$ in [6]. Then one can obtain the $L^{\infty}$ estimate as in Lemma 2.1.

Step 2: Oscillation estimates.

This is obtained in Lemma 3.6. We shall focus on this parts in the following discussions.

Step 3: Hölder regularity. 
This is followed by the oscillation estimated by a standard argument.

Now we turn to the proof of main results. We may consider the local estimate at a ball centered at $(0,0)$, since the equation (1.4) is invariant under the left translation when $a$ is constant. We follow the same route as [10], [7] and [8]. For convenience, we consider the estimates in the following cube, instead of $\mathcal{B}_{r}^{-}$,

$$
\mathcal{C}_{r}^{-}=\left\{(x, y, t)\left|\quad-r^{2} \leq t<0,\right| x|\leq r,| y \mid \leq(2 r)^{3}\right\}
$$

Let

$$
K_{r}=\left\{(x, y)|| x|\leq r,| y \mid \leq(2 r)^{3}\right\} .
$$

Let $0<\alpha, \beta<1$ be constants, for fixed $t$ and $h$, we denote

$$
\mathcal{N}_{t, h}=\left\{(x, y) \mid(x, y) \in K_{\beta r}, u(x, y, t) \geq h\right\}
$$

We sometimes abuse the notations of $\mathcal{B}_{r}^{-}$and $\mathcal{C}_{r}^{-}$, since there are equivalent.

Lemma 3.1 Suppose that $u(x, t) \geq 0$ be a solution of equation (1.5) in $\mathcal{C}_{r}^{-}$ centered at $(0,0)$ and

$$
\operatorname{mes}\left\{(x, t) \in \mathcal{C}_{r}^{-}, \quad u \geq 1\right\} \geq \frac{1}{2} \operatorname{mes}\left(\mathcal{C}_{r}^{-}\right),
$$

then there exist constants $\alpha, \beta$ and $h_{1}, 0<\alpha, \beta, h_{1}<1$, where $h_{1}$ only depends on $\mu$, such that for almost all $t \in\left(-\alpha r^{2}, 0\right)$ and $0<h<h_{1}$

$$
\operatorname{mes}\left\{\mathcal{N}_{t, h}\right\} \geq \frac{1}{11} \operatorname{mes}\left\{K_{\beta r}\right\} \text {. }
$$

Proof: Let

$$
v=\ln ^{+}\left(\frac{1}{u+h^{\frac{9}{8}}}\right),
$$

where $h$ is a constant, $0<h<1$, to be determined later. Then $v$ at points where $v$ is positive, satisfies

$$
\partial_{x}\left(a(x, y, t) \partial_{x} v\right)-a\left(\partial_{x} v\right)^{2}+b_{0}(x, y, t) \partial_{x} v+x \partial_{y} v-\partial_{t} v=0
$$


Let $\eta(s)$ be a smooth cut-off function so that

$$
\begin{gathered}
\eta(s)=1, \quad \text { for } \quad s<\beta r, \\
\eta(s)=0, \quad \text { for } \quad s \geq r .
\end{gathered}
$$

Moreover, $0 \leq \eta \leq 1$ and $\left|\eta^{\prime}\right| \leq \frac{2}{(1-\beta) r}$.

Multiplying $\eta(|x|)^{2}$ to (3.1) and integrating by parts on $K_{r} \times(\tau, t)$

$$
\begin{aligned}
& \int_{K_{\beta r}} v(x, y, t) d x d y+\int_{\tau}^{t} \int_{K_{r}} \eta^{2} a\left|\partial_{x} v\right|^{2} d x d y d s \\
\leq & \int_{\tau}^{t} \int_{K_{r}} \eta^{2}\left(\partial_{x}\left(a(x, y, t) \partial_{x} v\right)+b_{0}(x, y, t) \partial_{x} v+x \partial_{y} v\right) d x d y d s \\
& +\int_{K_{r}} v(x, y, \tau) d x d y \\
\leq & \frac{C(\mu)}{\beta^{4}(1-\beta)^{2}}\left|K_{\beta r}\right|+\int_{\tau}^{t} \int_{K_{r}}\left(\frac{1}{2} \eta^{2} a\left|\partial_{x} v\right|^{2}+x \partial_{y} v \eta^{2}\right) d x d y d s \\
& +\int_{K_{r}} v(x, y, \tau) d x d y, \quad \text { a.e. } \quad \tau, t \in\left(-r^{2}, 0\right) .
\end{aligned}
$$

Then

$$
\begin{aligned}
\left|\int_{K_{r}} x \partial_{y} v \eta^{2}\right| & =\left|\int_{|x| \leq r} x v \eta^{2}\right|_{y=-8 \beta^{3} r^{3}}^{8 \beta^{3} r^{3}} d x \mid \\
& \leq \frac{1}{4} r^{-2} \beta^{-4}\left|K_{\beta r}\right| \ln \left(h^{-\frac{9}{8}}\right) .
\end{aligned}
$$

Integrating by t to $I_{B}$, we have

$$
\left|\int_{\tau}^{t} \int_{K_{r}} x \partial_{y} v \eta^{2}\right| \leq \frac{1}{4} \beta^{-4}\left|K_{\beta r}\right| \ln \left(h^{-\frac{9}{8}}\right) .
$$

We shall estimate the measure of the set $\mathcal{N}_{t, h}$. Let

$$
\nu(t)=\operatorname{mes}\left\{(x, y) \mid \quad(x, y) \in K_{r}, u(x, y, t) \geq 1\right\} .
$$

By our assumption, for $0<\alpha<\frac{1}{2}$

$$
\frac{1}{2} r^{2} \operatorname{mes}\left(K_{r}\right) \leq \int_{-r^{2}}^{0} \nu(t) d t=\int_{-r^{2}}^{-\alpha r^{2}} \nu(t) d t+\int_{-\alpha r^{2}}^{0} \nu(t) d t,
$$


that is

$$
\int_{-r^{2}}^{-\alpha r^{2}} \nu(t) d t \geq\left(\frac{1}{2}-\alpha\right) r^{2} \operatorname{mes}\left(K_{r}\right)
$$

then there exists a $\tau \in\left(-r^{2},-\alpha r^{2}\right)$, such that

$$
\mu(\tau) \geq\left(\frac{1}{2}-\alpha\right)(1-\alpha)^{-1} \operatorname{mes}\left(K_{r}\right) .
$$

By noticing $v=0$ when $u \geq 1$, we have

$$
\int_{K_{r}} v(x, y, \tau) d x d y \leq \frac{1}{2}(1-\alpha)^{-1} m e s\left(K_{r}\right) \ln \left(h^{-\frac{9}{8}}\right) .
$$

Now we choose $\alpha$ (near zero), and $\beta$ (near one), such that

$$
\frac{1}{4 \beta^{4}}+\frac{1}{2 \beta^{4}(1-\alpha)} \leq \frac{4}{5}
$$

and fix them from now on.

By (3.2), (3.3), (3.4) and (3.5), we deduce

$$
\begin{aligned}
& \int_{K_{\beta r}} v(x, y, t) d x d y \\
& \leq\left[\frac{C(\mu)}{\beta^{4}(1-\beta)^{2}}+\frac{4}{5} \ln \left(h^{-\frac{9}{8}}\right)\right] \operatorname{mes}\left(K_{\beta r}\right) .
\end{aligned}
$$

When $(x, y) \notin \mathcal{N}_{t, h}$, we have

$$
\ln \left(\frac{1}{2 h}\right) \leq \ln ^{+}\left(\frac{1}{h+h^{\frac{9}{8}}}\right) \leq v,
$$

then

$$
\ln \left(\frac{1}{2 h}\right) m e s\left(K_{\beta r} \backslash \mathcal{N}_{t, h}\right) \leq \int_{K_{\beta r}} v(x, y, t) d x d y .
$$

Since

$$
\frac{C+\frac{4}{5} \ln \left(h^{-\frac{9}{8}}\right)}{\ln \left(h^{-1}\right)} \longrightarrow \frac{9}{10}, \quad \text { as } \quad h \rightarrow 0,
$$

then there exists constant $h_{1}$ such that for $0<h<h_{1}$ and $t \in\left(-\alpha r^{2}, 0\right)$

$$
\operatorname{mes}\left(K_{\beta r} \backslash \mathcal{N}_{t, h}\right) \leq \frac{10}{11} \operatorname{mes}\left(K_{\beta r}\right) .
$$


Then we proved our lemma.

Let $\chi(s)$ be a $C^{\infty}$ smooth function given by

$$
\begin{array}{ll}
\chi(s)=1 & \text { if } \quad s \leq \theta^{\frac{1}{6}} r, \\
\chi(s)=0 & \text { if } \quad s>r,
\end{array}
$$

where $\theta>0$ is a constant, to be determined in Lemma 3.4, and $\theta^{\frac{1}{6}}<\frac{1}{2}$. Moreover, we assume that

$$
0 \leq-\chi^{\prime}(s) \leq \frac{2}{\left(1-\theta^{\frac{1}{6}}\right) r}, \quad\left|\chi^{\prime \prime}(s)\right| \leq \frac{C}{r^{2}},
$$

and for any $\beta_{1}, \beta_{2}$, with $\theta^{\frac{1}{6}}<\beta_{1}<\beta_{2}<1$, we have

$$
\left|\chi^{\prime}(s)\right| \geq C\left(\beta_{1}, \beta_{2}\right) r^{-1}>0,
$$

if $\beta_{1} r \leq s \leq \beta_{2} r$.

For $(x, y) \in R^{2}, t \leq 0$, we set

$$
\mathcal{Q}=\left\{(x, y, t)\left|-r^{2} \leq t \leq 0,\right| x\left|\leq \frac{r}{\theta},\right| y \mid \leq \frac{r^{3}}{\theta}\right\} .
$$

We define the cut off functions by

$$
\begin{gathered}
\phi_{0}(x, y, t)=\chi\left(\left[\theta^{2} y^{2}-6 \operatorname{tr}^{4}\right]^{\frac{1}{6}}\right), \\
\phi_{1}(x, y, t)=\chi(\theta|x|), \\
\phi(x, y, t)=\phi_{0} \phi_{1} .
\end{gathered}
$$

Lemma 3.2 By the definition of $\phi$ and the above arguments, we have

$$
\left(x \partial_{y}-\partial_{t}\right) \phi_{0}(z) \leq 0, \quad \text { for } \quad \mathrm{z} \in \mathcal{Q} \text {. }
$$

And since $\theta^{\frac{1}{6}}<\frac{1}{2}$, we have

(1) $\phi(z) \equiv 1$, in $\mathcal{B}_{\theta r}^{-}$, 
(2) $\operatorname{supp} \phi \bigcap\left\{(\mathrm{x}, \mathrm{y}, \mathrm{t}) \mid(\mathrm{x}, \mathrm{y}) \in \mathrm{R}^{2}, \mathrm{t} \leq 0\right\} \subset \mathcal{Q}$,

(3) there exists a constant $\alpha_{1}, 0<\alpha_{1}<\min \left\{\alpha, \frac{1}{12}\right\}$, such that

$$
\left\{(x, y, t) \mid-\alpha_{1} r^{2} \leq t<0,(x, y) \in K_{\beta r}\right\} \subseteq \operatorname{supp} \phi,
$$

moreover, $0<\phi_{0}(z)<1$, for $z \in\left\{(x, y, t) \mid-\alpha_{1} r^{2} \leq t \leq-\theta r^{2},(x, y) \in K_{\beta r}\right\}$.

Proof: By the definition of $\phi_{0}$, we attain

$$
\left(x \partial_{y}-\partial_{t}\right) \phi_{0}=\chi^{\prime}\left(\left[\theta^{2} y^{2}-6 t r^{4}\right]^{\frac{1}{6}}\right) \frac{1}{6}\left[\theta^{2} y^{2}-6 t r^{4}\right]^{-\frac{5}{6}}\left[6 r^{4}+2 \theta^{2} x y\right] \leq 0 .
$$

When $\theta<\frac{1}{6}$, we can check that obviously (1) holds. We notice that either $|x| \geq \frac{r}{\theta}$, or $|y| \geq \frac{r^{3}}{\theta}$, or $t \leq-r^{2}$, then $\phi$ vanishes, hence we obtain (2). When $(x, y) \in K_{\beta r}$, then $\phi_{1}>0$ and we can choose $\theta<\frac{1}{64}$ and $t$ small, for example, $t>-\alpha_{1} r^{2}$, such that $\theta^{2} y^{2}-6 t r^{4}<r^{6}$, then we obtain (3).

Now we have the following Poincaré's type inequality.

Lemma 3.3 Let $w$ be a non-negative weak sub-solution of (1.5) in $\mathcal{B}_{1}^{-}$. Then there exists an absolute constant $C$, such that for $r<\theta<1$

$$
\int_{\mathcal{B}_{\theta r}^{-}}\left(w(z)-I_{0}\right)_{+}^{2} \leq C \theta^{2} r^{2} \int_{\mathcal{B}_{\frac{r}{\theta}}^{-}}\left|\partial_{x} w\right|^{2},
$$

where $I_{0}=\sup _{\mathcal{B}_{\theta r}^{-}} I_{1}(z)$, and

$$
I_{1}(z)=\int_{\mathcal{B}_{\frac{r}{\theta}}^{-}}\left[-\Gamma(z, \zeta) w(\zeta)\left(\xi \partial_{\eta}-\partial_{\tau}\right) \phi(\zeta)-\partial_{\xi}^{2} \phi(\zeta) \Gamma(z, \zeta) w(\zeta)\right] d \zeta,
$$

where $\Gamma$ is the fundamental solution of $L_{0}$, and $\phi$ is given by (3.7).

Proof: We represent $w$ in terms of the fundamental solution of $\Gamma$, i.e.

$$
\varphi(z)=-\int_{R^{2+1}} \Gamma(z, \zeta) L_{0} \varphi(\zeta) d \zeta, \quad \forall \varphi \in C_{0}^{\infty}\left(R^{2+1}\right) .
$$


By an approximation of $\phi$ and integrating by parts, for $z \in \mathcal{B}_{\theta r}^{-}$, we have

$$
\begin{aligned}
w(z) & =\int_{\mathcal{B}_{\frac{r}{\theta}}^{-}}\left[\left\langle\partial_{\xi}(w \phi)(\zeta), \partial_{\xi} \Gamma(z, \zeta)\right\rangle-\Gamma(z, \zeta)\left(\xi \partial_{\eta}-\partial_{\tau}\right)(w \phi)(\zeta)\right] d \zeta \\
& =I_{1}(z)+I_{2}(z)+I_{3}(z)
\end{aligned}
$$

where

$$
\begin{gathered}
I_{1}(z)=\int_{\mathcal{B}_{\frac{r}{\theta}}^{-}}\left[-\Gamma(z, \zeta) w\left(\xi \partial_{\eta}-\partial_{\tau}\right) \phi+\left\langle\partial_{\xi} \phi, \partial_{\xi} \Gamma(z, \zeta)\right\rangle w+\Gamma(z, \zeta)\left\langle\partial_{\xi} w, \partial_{\xi} \phi\right\rangle\right] d \zeta, \\
I_{2}(z)=\int_{\mathcal{B}_{\frac{r}{\theta}}}\left[\left\langle(1-a) \partial_{\xi} w, \partial_{\xi} \Gamma(z, \zeta)\right\rangle \phi-\Gamma(z, \zeta)\left\langle(a+1) \partial_{\xi} w, \partial_{\xi} \phi\right\rangle\right. \\
\left.+\Gamma(z, \zeta) \phi b_{0} \partial_{\xi} w\right] d \zeta=I_{21}+I_{22}+I_{23},
\end{gathered}
$$

and

$$
I_{3}(z)=\int_{\mathcal{B}_{\frac{r}{\theta}}}\left[\left\langle a \partial_{\xi} w, \partial_{\xi}(\Gamma(z, \zeta) \phi)\right\rangle-\Gamma(z, \zeta) \phi\left(b_{0} \partial_{\xi}+\xi \partial_{\eta}-\partial_{\tau}\right) w\right] d \zeta
$$

Note that $\operatorname{supp} \phi \bigcap\{\tau \leq 0\} \subset \mathcal{Q} \subset \overline{\mathcal{B}_{\frac{r}{\theta}}^{-}}, z \in \mathcal{B}_{\theta r}^{-}$and $\left\langle\partial_{\xi} \phi, \partial_{\xi} \Gamma(z, \zeta)\right\rangle$ vanishes in a small neighborhood of $z$. Integrating by parts we obtain $I_{1}(z)$ as in (3.10).

From our assumption, $w$ is a weak sub-solution of (1.5), and $\phi$ is a test function of this semi-cylinder. In fact, we let

$$
\tilde{\chi}(\tau)= \begin{cases}1 & \tau \leq 0 \\ 1-n \tau & 0 \leq \tau \leq 1 / n \\ 0 & \tau \geq 1 / n\end{cases}
$$

Then $\tilde{\chi}(\tau) \phi \Gamma(z, \zeta)$ can be a test function (see [6]). As $n \rightarrow \infty$, we obtain $\phi \Gamma(z, \zeta)$ as a legitimate test function, and $I_{3}(z) \leq 0$. Then in $\mathcal{B}_{\theta r}^{-}$,

$$
0 \leq\left(w(z)-I_{0}\right)_{+} \leq I_{2}(z)
$$


By Corollary 2.1 we have

$$
\left\|I_{21}\right\|_{L^{2}\left(\mathcal{B}_{\theta r}^{-}\right)} \leq C \theta r\left\|I_{21}\right\|_{L^{3}\left(\mathcal{B}_{\theta r}^{-}\right)} \leq C \theta r\left\|\partial_{\xi} w\right\|_{L^{2}\left(\mathcal{B}_{\frac{r}{\theta}}^{-}\right)} .
$$

Similarly

$$
\left\|I_{2 i}\right\|_{L^{2}\left(\mathcal{B}_{\theta r}^{-}\right)} \leq C \theta^{2} r\left\|\partial_{\xi} w\right\|_{L^{2}\left(\mathcal{B}_{\frac{r}{\theta}}^{-}\right)}, \quad i=2,3
$$

then we proved our lemma.

Now we apply Lemma 3.3 to the function $w=\ln ^{+} \frac{h}{u+h^{\frac{9}{8}}}$. If $u$ is a weak solution of (1.5), then $w$ is a weak sub-solution. We estimate the value of $I_{0}$.

Lemma 3.4 Under the assumptions of Lemma 3.3, there exist constants $\theta$, $\lambda_{0}, \lambda_{0}<1$ only depends on constants $\alpha$ and $\beta$, such that for $r<\theta$

$$
\left|I_{0}\right| \leq \lambda_{0} \ln \left(h^{-\frac{1}{8}}\right)
$$

Proof: We first come to estimate the second term of $I_{1}(z)$ and as before, denote $z=(x, y, t)$ and $\zeta=(\xi, \eta, \tau)$. Note $z \in \mathcal{B}_{\theta r}^{-}$, we have

$$
\begin{aligned}
& \int_{\mathcal{B}_{\frac{r}{\theta}}^{-}}\left[\left|\partial_{\xi}^{2} \phi(\zeta)\right| \Gamma(z, \zeta) w\right] d \zeta \\
\leq & r^{2} \sup _{\xi \in \operatorname{supp}\left(\partial_{\xi} \phi\right)}\left|\partial_{\xi}^{2} \phi\right|(\zeta) \ln \left(h^{-\frac{1}{8}}\right) . \quad(B y
\end{aligned}
$$

We only need to estimate $\left|\partial_{\xi}^{2} \phi_{1}\right|$. Since

$$
\left|\partial_{\xi} \phi_{1}\right|=\left|\theta \chi^{\prime}(\theta|\xi|) \partial_{\xi}\right| \xi|| \leq 4 \theta r^{-1}, \quad\left|\partial_{\xi}^{2} \phi_{1}\right| \leq C \theta^{2} r^{-2} \text {. }
$$

Hence

$$
\left|\int_{\mathcal{B}_{\frac{r}{\theta}}^{-}}\left[-\partial_{\xi}^{2} \phi \Gamma(z, \zeta) w\right] d \zeta\right| \leq C_{3} \theta^{2} \ln \left(h^{-\frac{1}{8}}\right)
$$

where $C_{3}$ is an absolute constant.

Now we let $w \equiv 1$, then for $z \in \mathcal{B}_{\theta r}^{-},(3.11)$ gives

$$
1=\int_{\mathcal{B}_{\frac{r}{\theta}}^{-}}\left[-\phi_{1} \Gamma(z, \zeta)\left(\xi \partial_{\eta}-\partial_{\tau}\right) \phi_{0}\right] d \zeta+\int_{\mathcal{B}_{\frac{r}{\theta}}^{-}}\left[-\partial_{\xi}^{2} \phi(\zeta) \Gamma(z, \zeta)\right] d \zeta
$$


By (3.8) in Lemma 3.2, we know that

$$
-\phi_{1} \Gamma(z, \zeta)\left(\xi \partial_{\eta}-\partial_{\tau}\right) \phi_{0} \geq 0
$$

We only need to prove $-\phi_{1} \Gamma(z, \zeta)\left(\xi \partial_{\eta}-\partial_{\tau}\right) \phi_{0}$ has a positive lower bound in a domain which $w$ vanishes, and this bound independent of $r$ and small $\theta$. So we can find a $\lambda_{0}, 0<\lambda_{0}<1$, such that this lemma holds.

For $z \in B_{\theta r}^{-}$, set

$$
\zeta \in Z=\left\{(\xi, \eta, \tau) \mid-\alpha_{1} r^{2} \leq \tau \leq-\frac{\alpha_{1}}{2} r^{2},(\xi, \eta) \in K_{\beta r}, w(\xi, \eta, \tau)=0\right\},
$$

then by Lemma $3.1,|Z|=C\left(\alpha_{1}, \beta\right) r^{6}$.

We note that when $\zeta=(\xi, \eta, \tau) \in Z$ and $\theta<\frac{1}{64}, w(\zeta)=0, \phi_{1}(\zeta)=1$, and

$$
\left|\chi^{\prime}\left(\left[\theta^{2} y^{2}-6 t r^{4}\right]^{\frac{1}{6}}\right)\right| \geq C\left(\alpha_{1}\right) r^{-1}>0
$$

Consequently

$$
\begin{aligned}
& \int_{Z}\left[-\phi_{1} \Gamma(z, \zeta)\left(\xi \partial_{\eta}-\partial_{\tau}\right) \phi_{0}\right] d \zeta \\
& =-\int_{Z} \phi_{1} \Gamma(z, \zeta) \chi^{\prime}\left(\left[\theta^{2} \eta^{2}-6 \tau r^{4}\right]^{\frac{1}{6}}\right) \frac{1}{6}\left[\theta^{2} \eta^{2}-6 \tau r^{4}\right]^{-\frac{5}{6}}\left[6 r^{4}+2 \theta^{2} \xi \eta\right] d \zeta \\
& \geq C\left(\alpha_{1}\right) \int_{Z} r^{-2} \Gamma\left(\zeta^{-1} \circ z, 0\right) d \zeta=C(\alpha, \beta)=C_{4}>0,
\end{aligned}
$$

where we have used $\Gamma(z, \zeta) \geq C r^{-4}$, as $\tau \leq-\frac{\alpha_{1}}{2} r^{2}$ and $z \in B_{\theta r}^{-}$. In fact, by (2.2) one can obtain this result easily.

By (3.14) and

$$
I_{0}(z)=\sup _{\mathcal{B}_{\theta r}^{-}} \int_{\mathcal{B}_{\frac{r}{\theta}}^{-} \backslash Z}\left[-\Gamma(z, \zeta) w(\zeta)\left(\xi \partial_{\eta}-\partial_{\tau}\right) \phi(\zeta)-\partial_{\xi}^{2} \phi(\zeta) \Gamma(z, \zeta) w(\zeta)\right] d \zeta
$$

we have

$$
\left|I_{0}\right| \leq\left(1-C_{4}+C_{3} \theta^{2}\right) \ln \left(h^{-\frac{1}{8}}\right)+C_{3} \theta^{2} \ln \left(h^{-\frac{1}{8}}\right) .
$$


We can choose a small $\theta$ which is fixed from now on, such that $\left|I_{0}\right| \leq$ $\lambda_{0} \ln \left(h^{-\frac{1}{8}}\right)$, where $0<r<\theta$, and $0<\lambda_{0}<1$ which only depends on $\alpha$ and $\beta$.

The following two Lemmas are similar to those in [8], we give them for completeness.

Lemma 3.5 Suppose that $u \geq 0$ is a solution of equation (1.5) in $\mathcal{B}_{r}^{-}$centered at $(0,0)$ and mes $\left\{(x, y, t) \in \mathcal{B}_{r}^{-}, u \geq 1\right\} \geq \frac{1}{2} \operatorname{mes}\left(\mathcal{B}_{r}^{-}\right)$. Then there exist constants $\theta$ and $h_{0}, 0<\theta, h_{0}<1$ which only depend on $\lambda_{0}$ and $\mu$, such that

$$
u(x, y, t) \geq h_{0} \quad \text { in } \quad \mathcal{B}_{\theta r}^{-}
$$

Proof: We consider

$$
w=\ln ^{+}\left(\frac{h}{u+h^{\frac{9}{8}}}\right),
$$

for $0<h<1$, to be decided. By applying Lemma 3.3 to $w$, we have

$$
f_{\mathcal{B}_{\theta r}^{-}}\left(w-I_{0}\right)_{+}^{2} \leq C \frac{\theta r^{2}}{\left|\mathcal{B}_{\theta r}^{-}\right|} \int_{\mathcal{B}_{r}^{-}}\left|\partial_{x} w\right|^{2} .
$$

Let $\tilde{u}=\frac{u}{h}$, then $\tilde{u}$ satisfies the conditions of Lemma 3.1. We can get similar estimates as (3.2), (3.3), (3.4) and (3.5), hence we have

$$
\begin{aligned}
& C \frac{\theta r^{2}}{\left|\mathcal{B}_{\theta r}^{-}\right|} \int_{\mathcal{B}_{r}^{-}}\left|\partial_{x} w\right|^{2} \\
& \leq C(\mu) \frac{\theta r^{2}}{\left|\mathcal{B}_{\theta r}^{-}\right|}\left[\frac{C(\mu)}{\beta^{4}(1-\beta)^{2}}+\frac{4}{5} \ln \left(h^{-\frac{1}{8}}\right)\right] \operatorname{mes}\left(K_{\beta^{-1} r}\right) \\
& \leq C(\theta, \mu, \beta) \ln \left(h^{-\frac{1}{8}}\right)
\end{aligned}
$$

where $\theta$ has been chosen. By $L^{\infty}$ estimate, there exists a constant, still denoted by $\theta$, such that for $z \in \mathcal{B}_{\theta r}^{-}$,

$$
w-I_{0} \leq C(\mu, \beta)\left(\ln \left(h^{-\frac{1}{8}}\right)\right)^{\frac{1}{2}} .
$$


Therefore we may choose $h_{0}$ small enough, so that

$$
C(\mu, \beta)\left(\ln \left(\frac{1}{h_{0}^{\frac{1}{8}}}\right)\right)^{\frac{1}{2}} \leq \ln \left(\frac{1}{2 h_{0}^{\frac{1}{8}}}\right)-\lambda_{0} \ln \left(\frac{1}{h_{0}^{\frac{1}{8}}}\right) .
$$

Then (3.13) and (3.19) derive

$$
\max _{\mathcal{B}_{\theta r}^{-}} \frac{h_{0}}{u+h_{0}^{\frac{9}{8}}} \leq \frac{1}{2 h_{0}^{\frac{1}{8}}},
$$

which implies $\min _{\mathcal{B}_{\theta r}^{-}} u \geq h_{0}^{\frac{9}{8}}$, then we finished the proof of this Lemma.

Lemma 3.6 Suppose that $u$ is a weak solution of equation (1.5) in $\mathcal{B}_{r}^{-}$, then exists constant $h_{2}, 0<h_{2}<1$, such that

$$
O s c_{\mathcal{B}_{\theta r}^{-}} u \leq h_{2} O s c_{\mathcal{B}_{r}^{-}} u
$$

where $\theta$ is given in Lemma 3.5 .

Proof: We may assume that $M=\max _{\mathcal{B}_{r}^{-}}(+u)=\max _{\mathcal{B}_{r}^{-}}(-u)$, otherwise we replace $u$ by $u-c$, since $u$ is bounded locally. Then either $1+\frac{u}{M}$ or $1-\frac{u}{M}$ satisfies the assumption of Lemma 3.5, and we suppose $1+\frac{u}{M}$ does, thus Lemma 3.5 implies that there exists $h_{0}>0$ such that

$$
\inf _{\mathcal{B}_{\theta r}^{-}}\left(1+\frac{u}{M}\right) \geq h_{0}
$$

that is $u \geq M\left(h_{0}-1\right)$, then

$$
O s c_{\mathcal{B}_{\theta r}^{-}} u \leq M-M\left(h_{0}-1\right) \leq\left(1-\frac{h_{0}}{2}\right) O s c_{\mathcal{B}_{r}^{-}} u,
$$

where we can let $h_{2}=\left(1-\frac{h_{0}}{2}\right)$.

Proof of Theorem 2.2. By the standard regularity arguments, for example, see Chapter 8 in [4], we can obtain the result near point $(0,0)$. By the left invariant translation group action, we know that $u$ is $C^{\alpha}$ in the interior. 


\section{Proof of Main Theorem}

By Theorem 2.2 and a variable transformation (see [9]), we can prove Theorem 1.1.

Proof: Since $\partial_{x} b(x, y, t) \neq 0$, let $b=\xi, y=\eta$, and $t=\tau$, then

$$
\frac{\partial}{\partial x}=\frac{\partial b}{\partial x} \frac{\partial}{\partial \xi}, \quad \frac{\partial}{\partial y}=\frac{\partial}{\partial \eta}+\frac{\partial b}{\partial y} \frac{\partial}{\partial \xi}, \quad \frac{\partial}{\partial t}=\frac{\partial}{\partial \tau}+\frac{\partial b}{\partial t} \frac{\partial}{\partial \xi},
$$

and the equations (1.1) can be written as

$$
\frac{\partial b}{\partial x} \frac{\partial}{\partial \xi}\left(a \frac{\partial b}{\partial x} \frac{\partial}{\partial \xi} u\right)+\left(b_{0} \frac{\partial b}{\partial x}+\xi \frac{\partial b}{\partial y}-\frac{\partial b}{\partial t}\right) \frac{\partial}{\partial \xi} u+\xi \partial_{\eta} u-\partial_{\tau} u=0 .
$$

From the implicit function theorem, we know $(x, y, t): \rightarrow(\xi, \eta, \tau)$ is a $C^{2}$ diffeomorphism, and $\frac{\partial b}{\partial x}=\left(\frac{\partial x(\xi, \eta, \tau)}{\partial \xi}\right)^{-1}$, hence the above equation can attain

$$
\frac{\partial}{\partial \xi}\left(\frac{\partial b}{\partial x} a \frac{\partial b}{\partial x} \frac{\partial}{\partial \xi} u\right)+\left[a \frac{\partial^{2} x}{\partial \xi^{2}}\left(\frac{\partial b}{\partial x}\right)^{3}+b_{0} \frac{\partial b}{\partial x}+\xi \frac{\partial b}{\partial y}-\frac{\partial b}{\partial t}\right] \frac{\partial}{\partial \xi} u+\xi \partial_{\eta} u-\partial_{\tau} u=0,
$$

that is

$$
\frac{\partial}{\partial \xi}\left(\tilde{a} \frac{\partial}{\partial \xi} u\right)+\tilde{b_{0}} \frac{\partial}{\partial \xi} u+\xi \partial_{\eta} u-\partial_{\tau} u=0
$$

where $\tilde{a}=\frac{\partial b}{\partial x} a \frac{\partial b}{\partial x}$, and $\tilde{b_{0}}=a \frac{\partial^{2} x}{\partial \xi^{2}}\left(\frac{\partial b}{\partial x}\right)^{3}+b_{0} \frac{\partial b}{\partial x}+\xi \frac{\partial b}{\partial y}-\frac{\partial b}{\partial t}$.

In a fixed bounded domain, $\tilde{a} \in L^{\infty}$ and $\tilde{b_{0}} \in L^{\infty}$, by Theorem 2.2 the weak solution of (4.3) is Hölder continuous.

We give an immediate corollary. Let $x=\left(x_{1}, \ldots, x_{m}\right), y=\left(y_{1}, \ldots, y_{n}\right)$ and $m \geq n$.

$L u \equiv \sum_{i, j=1}^{m} \partial_{x_{i}}\left(a_{i j}(x, y, t) \partial_{x_{j}} u\right)+\sum_{k=1}^{m} b_{0}^{k}(x, y, t) \partial_{x_{k}} u+\sum_{l=1}^{n} b_{l}(x, y, t) \partial_{y_{l}} u-\partial_{t} u=0$,

and we assume: 
[H.1] the coefficients $a_{i j}, 1 \leq i, j \leq m$, are real valued, measurable functions of $(x, t)$. Moreover, $a_{i j}=a_{j i} \in L^{\infty}\left(R^{m+n+1}\right)$ and there exists a $\mu>0$ such that

$$
\mu \sum_{i=1}^{m} \xi_{i}^{2} \leq \sum_{i, j=1}^{m} a_{i j}(x, y, t) \xi_{i} \xi_{j} \leq \frac{1}{\mu} \sum_{i=1}^{m} \xi_{i}^{2}
$$

for every $(x, y, t) \in R^{m+n+1}$, and $\xi \in R^{m}$.

[H.2] $b_{0}^{j} \in L^{\infty}(\Omega), b_{l} \in C^{2}(\Omega)$, and $\left|b_{0}^{j}\right|_{\infty},\left|b_{l}\right|_{C^{2}} \leq \frac{1}{\mu}$, where $j=1, \ldots, m$ and $l=1, \ldots, n$. There exists $i_{1}, \cdots, i_{n}$, such that $\frac{\partial\left(b_{1}, \ldots, b_{n}\right)}{\partial\left(x_{i_{1}}, \ldots, x_{i_{n}}\right)} \neq 0$, where $i_{j} \in\{1, \ldots, m\}, j=1, \ldots, n$ and $i_{1}<\cdots<i_{n}$.

Corollary 4.1 Under the assumption [H.1] and [H.2], the weak solutions of (4.4) are Hölder continuous.

\section{References}

[1] C. Cinti, A. Pascucci and S. Polidoro, Pointwise estimates for solutions to a class of non-homogenous Kolmogorov equations, Mathematische Annalen, Volume 340, n.2, pp.237-264, (2008).

[2] C. Cinti and S. Polidoro, Pointwise local estimates and Gaussian upper bounds for a class of uniformly subelliptic ultraparabolic operators, J. Math. Anal. Appl. 338, 946-969 (2008).

[3] G. B. Folland, Subellitic estimates and function space on nilpotent Lie groups, Ark. Math., 13 (2): 161-207, (1975).

[4] D. Gilberg, and N. S. Trudinger: Elliptic Partial Differential Equations of Second Order, 3nd ed, Springer-Verlag, Berlin, 2001. 
[5] E. Lanconelli and S. Polidoro, On a class of hypoelliptic evolution operaters, Rend. Sem. Mat. Univ. Politec. Torino, 52,1 , 29-63, (1994).

[6] A. Pascucci and S. Polidoro, The moser's iterative method for a class of ultraparabolic equations, Commun. Contemp. Math. Vol. 6, No. 3, 395-417 (2004).

[7] W. Wang and L. Zhang, The $C^{\alpha}$ regularity of a class of nonhomogeneous ultraparabolic equations, arXiv:math.AP/0711.3411.

[8] W. Wang and L. Zhang, The $C^{\alpha}$ regularity of a class of hypoelliptic ultraparabolic equations, arXiv:math.AP/0804.4358v2.

[9] M. Weber, The fundamental solution of a degenerate partial differential equation of parabolic type, Trans. Amer. Math. Soc., 71, 24-37 (1951).

[10] L. Zhang, The $C^{\alpha}$ reglarity of a class of ultraparabolic equations, arXiv:math.AP/0510405v2

[11] L. Zhang, The $C^{\alpha}$ reglarity of a class of ultraparabolic equations, AMS/IP Studies in Adv. Math., vol 42, 619-622 (2008). 